\title{
Loss of function of SWI/SNF chromatin remodeling genes leads to genome instability of human lung cancer
}

\author{
HAI-TAO HUANG, SHAO-MU CHEN, LIANG-BIN PAN, JIE YAO and HAI-TAO MA \\ Department of Cardiothoracic Surgery, The First Affiliated Hospital of Soochow University, \\ Suzhou, Jiangsu 215006, P.R. China
}

Received August 19, 2014; Accepted September 18, 2014

DOI: $10.3892 /$ or.2014.3584

\begin{abstract}
SWI/SNF chromatin remodeling complexes are frequently mutated in a variety of human cancers. We investigated the mutation incidence and the role of $\mathrm{mSWI} / \mathrm{SNF}$ (BAF) complexes in human lung cancer. In the present study, we analyzed somatic mutations of BAF complexes and other driver mutated genes of lung carcinoma deposited in the Catalogue of Somatic Mutations in Cancer (COSMIC) database. BAF complexes were mutated in 282 of 803 (35.12\%) lung carcinoma samples analyzed, ranking second to TP53. Significantly, BAF-mutated samples exhibited more genomic mutations than BAF wild-type ones. Moreover, a significant positive correlation existed between the BAF mutations and overall genomic mutations in these lung carcinoma samples $(\mathrm{P}<0.001$, Pearson's correlation analysis). Specifically, the mutant-typing of 6 BAF genes, SMARCA4, ARID2, ARIDIB, $B C L 11 A, B C L 11 B$ and $B R D 9$ was associated with more overall mutations in the lung carcinoma samples. A mutation reporter system was developed by means of the establishment of stable cell sublines with slippage-luciferase transcript in a lung adenocarcinoma cell line, Calu-3. SMARCA4, the most frequently mutated BAF gene in lung cancer, was stably knocked down by pSUPER constructs carrying short hairpin RNA (shRNA). Mutation ratios determined from the mutation reporters of Calu-3 cells were significantly increased upon stable SMARCA4 knockdown. We demonstrated that genetic mutations of BAF complexes lead to genome instability of
\end{abstract}

Correspondence to: Dr Hai-Tao Ma, Department of Cardiothoracic Surgery, The First Affiliated Hospital of Soochow University, 188 Shizi Street, Suzhou, Jiangsu 215006, P.R. China

E-mail:maht_sz215006@163.com

Abbreviations: ADC, adenocarcinoma; BMI, BAF mutation index; COSMIC, Catalogue of Somatic Mutations in Cancer; Luc, luciferase; NSCLC, non-small cell lung cancer; SCLC, small cell lung cancer; shRNA, short hairpin RNA; SCC, squamous cell carcinoma; WGS, whole genome sequencing

Key words: SWI/SNF, mutation reporter, lung cancer, genome instability, slippage-luciferase lung carcinoma. Therefore, BAF complexes play an important role in maintaining genome stability in human lung cancer.

\section{Introduction}

Lung cancer is the most common human cancer with a dismal prognosis, and it ranks highly in terms of cancerrelated mortality worldwide $(1,2)$. In China, lung cancer has replaced liver cancer as the leading cause of cancer-related deaths $(3,4)$. The two major types of lung cancers are small cell lung cancer (SCLC) accounting for $\sim 15 \%$ of primary lung cancers and non-small cell lung cancer (NSCLC), of which the most common subtypes are adenocarcinoma (ADC) and squamous cell carcinoma (SCC). As generally known, lung cancer, similar to many other cancers, arises as a result of the accumulation of genetic and epigenetic alterations, many of which ultimately confer growth and metastatic advantages upon the tumor cells $(5,6)$. The identification of genetic abnormalities of numerous key oncogenes and tumor suppressors, such as EGFR mutations, KRAS mutations and $p 53$ inactivated mutations, have improved our understanding of the molecular mechanisms involved in lung cancer development. Moreover, rapid technological developments in genome sequencing offer a more comprehensive and sophisticated genomic landscape of lung cancers. Among the somatic mutations recently identified in tumor specimens, somatic mutations in genes encoding chromatin remodeling proteins have frequently been identified in a variety of human cancers, including lung cancer $(7,8)$. Importantly, somatic mutations have been frequently detected in genes encoding the subunits of the switch/sucrose nonfermenting (SWI/SNF) complex in various cancers $(7,9)$. Inactivation mutations in several SWI/SNF subunits, such as BRG1/SMARCA4, ARIDIA and ARID2, have recently been identified in a significant proportion of lung tumors (10-12), indicating that SWI/SNF complexes represent a novel link between chromatin remodeling and tumor suppression in lung cancer.

SWI/SNF chromatin remodeling complexes, which use the energy of ATP hydrolysis to remodel nucleosomes and to mediate transcription, play critical roles in various biological processes including proliferation, differentiation and DNA repair $(7,9)$. Mammalian SWI/SNF (mSWI/SNF, also called BAF) complexes are polymorphic assemblies of at least 13 subunits encoded by 26 genes, making a 
widespread diversity of complexes with specialized function in specific tissues $(13,14)$. BAF complexes, similar to known tumor-suppressor TP53, are the most frequently and broadly mutated genes in human cancer (14).

To date, the genomic landscape of lung cancer which has been captured in several whole genome sequencing (WGS) projects has contributed to the comprehensive understanding of the genetic pathogenesis of human lung cancer. There is a large amount of information available in the published literature concerning genetic variants in human lung cancer. The Catalogue of Somatic Mutations in Cancer (COSMIC) database stores somatic mutations and associated information from human cancers extracted from the primary literature $(15,16)$. In the present study, we sought to determine the role of BAF complex mutations in lung cancer through analysis of the mutation incidence and evaluation of the mutation rate in an established mutation reporter of lung cancer cells. We utilized the COSMIC database to acquire the mutations of the 26 genes encoding BAF complexes in available tissue specimens and cell lines of lung cancer. Notably, our analysis showed that BAF complexes were mutated in 282 of $803(35.12 \%)$ tumor samples included, ranking second to TP53, and could contribute to the role of genome stability or DNA repair in lung cancer.

\section{Materials and methods}

Mutation profile analysis. The mutation information including mutations of the 26 BAF genes and the top- 10 frequently mutated genes in human lung carcinoma was obtained from the COSMIC (http://www.sanger.ac.uk/cosmic) database $(15,16)$. Each type of simple mutation (point mutation, small insertion and deletion) was represented by a specific mutation value, ' 0 ', without somatic mutation; ' 1 ', synonymous mutation; ' 2 ', missense mutation; ' 3 ', nonsense or frameshift mutation. The accumulated mutations of the 26 BAF genes in several lung carcinoma samples were then indicated by a defined BAF mutation index (BMI), equal to the sum of the mutation values from the $26 \mathrm{BAF}$ genes.

Construction of the slippage-luciferase vector. For the construction of the slippage-luciferase (slippage-Luc) vector, the luciferase-coding sequence with a mutated initiation codon (ATG to CTG) was first PCR-amplified from the pGL3 vector (Promega), and cloned into the BamHI and HindIII site of the pcDNA3.1/Hygro vector (Invitrogen). An ATG initiation codon followed with the oligonucleotides containing CA repeats leads to out of frame luciferase expression and was cloned into the XhoI and BamHI site of the recombinant pcDNA3.1/Hygro-Luc construct. The oligonucleotides containing CA repeats were synthesized as follows: $\mathrm{XhoI}$ ATG-(CA) $\left.{ }_{17}\left(5^{\prime} \text {-TCGAGCGCCATGGAA(CA) }\right)_{17} \mathrm{G}-3^{\prime}\right)$ and BamHI-(CA) $)_{17}\left(5^{\prime}-\right.$ GATCC(TG) ${ }_{17}$ TTCCATGGCGC-3'). The annealed oligonucleotides were then ligated into the $\mathrm{XhoI}$ and BamHI site of the modified pcDNA3.1/Hygro-Luc construct to acquire the slippage-Luc vector.

Establishment of the mutation reporter. The slippage-Luc vector was then linearized by $B g l \mathrm{II}$ digestion and transfected into Calu-3 cells using the Lipofectamine transfection reagent
(Invitrogen) according to the manufacturer's instructions. After $48 \mathrm{~h}$, the transfected cells were subsequently exposed to selection in medium containing $500 \mu \mathrm{g} / \mathrm{ml}$ Hygromycin (Clontech). The cells were fed with selective medium every 3-4 days until hygromycin-resistant colonies could be visible. Expanded colonies stably expressing the slip-luciferase transcripts were assessed by PCR.

pSUPER constructs for RNA interference against SMARCA4. For SMARCA4 knockdown, 4 fragments of sequences targeting against SMARCA4 were as follows: GCACCAGGAATACCTCAAT, GGTCAATGGTGTCCTC AAA, GGAGCACAAACGCATCAAT and GGCTCTGAGT ACTTCATCT, and their corresponding oligonucleotides for transcribing short hairpin RNA (shRNA) were synthesized. Then these annealed phosphorylated oligos were inserted into pSUPER carrying the neomycin-resistance gene, respectively; pSUPER carrying an irrelevant nucleotide not matching any human gene sequences was used as a negative control (pSUPER-shNC). RNAi knockdown efficiency of these pSUPER constructs against SMARCA4 were evaluated 3 days after transfection in the Calu-3 cell sublines. Stable SMARCA4 knockdown was identified from double stable colonies with hygromycin- and G418-resistance of Calu-3 cells.

Extraction of DNA and RNA. Genomic DNA was extracted from the stable cell lines using the DNeasy Tissue kit (Qiagen), and RNA was extracted using TRIzol ${ }^{\circledR}$ reagent (Invitrogen) according to the protocols recommended by the manufacturer, respectively. The total RNA was treated by RNase-free DNase I (Fermentas) to remove DNA contamination.

Reverse transcription-polymerase chain reaction (RT-PCR). The first-stand cDNA was transcribed by PrimerScript ${ }^{\mathrm{TM}} \mathrm{RT}$ reagent kit with gDNA Eraser (Takara). Amplified PCR products were observed by electrophoresis on a $2 \%$ agarose gel and visualized after staining with ethidium bromide. Real-time RT-PCR was performed using a CFX96 system (BioRad), and relative expression levels were calculated as $2^{-\Delta \Delta \mathrm{Ct}}$ according to the manufacturer's protocol. All experiments were performed in triplicate.

SDS-PAGE and western blot assay. Total protein extracts of stable cell lines were subjected to protein gel electrophoresis using $8 \%$ SDS-PAGE and were then transferred onto a nitrocellulose membrane (Amersham). After blocking with phosphate-buffered saline (PBS) containing 5\% nonfat milk, the membrane was incubated for immunoblotting assay with the SMARCA4 antibody (1:200 dilution, Santa Cruz) at $4^{\circ} \mathrm{C}$ overnight. The immunostaining signal was visualized with the Odyssey infrared imaging system (Li-Cor Biosciences).

Quantitative analysis for mutation rates. Luciferase activity was measured using the Luciferase reporter assay system (Promega) according to the instructions of the manufacturer. The experiments were performed at least 6 times, and the data were subjected to Luria-Delbrück fluctuation analyses $(17,18)$.

Statistical analysis. The two-tailed t-test was used to compare mutated genes or simple mutations, and the statis- 
Table I. Mutation frequencies of driver mutated genes in various types of lung carcinoma.

Frequency of mutation, mutated samples/total samples (\%)

\begin{tabular}{lrrrrr} 
& & \multicolumn{3}{c}{ Histological type } & \\
\cline { 2 - 4 } Mutated genes & \multicolumn{1}{c}{ Lung carcinoma } & \multicolumn{1}{c}{ ADC } & \multicolumn{1}{c}{ SCC } & \multicolumn{1}{c}{ SCLC } & P-value $^{\mathrm{a}}$ \\
\cline { 3 - 4 } BAF & $282 / 803(35.12)$ & $206 / 582(35.40)$ & $67 / 177(37.85)$ & $9 / 44(20.45)$ & 0.093 \\
TP53 & $1,423 / 3,142(45.29)$ & $715 / 1,858(38.48)$ & $530 / 1,040(50.96)$ & $178 / 244(72.95)$ & $<0.001$ \\
EGFR & $9,478 / 30,658(30.92)$ & $9,236 / 26,502(34.85)$ & $204 / 3,622(5.63)$ & $38 / 534(7.12)$ & $<0.001$ \\
KRAS & $2,254 / 13,545(16.64)$ & $2,131 / 10,778(19.77)$ & $116 / 2,290(5.07)$ & $7 / 477(1.47)$ & $<0.001$ \\
MLL3 & $133 / 848(15.68)$ & $96 / 609(15.76)$ & $33 / 180(18.33)$ & $4 / 59(6.78)$ & 0.106 \\
MLL2 & $85 / 846(10.05)$ & $44 / 609(7.22)$ & $35 / 180(19.44)$ & $6 / 57(10.53)$ & $<0.001$ \\
ZNF521 & $83 / 851(9.75)$ & $59 / 609(9.69)$ & $14 / 180(7.78)$ & $10 / 62(16.13)$ & 0.160 \\
NF1 & $110 / 1,208(9.11)$ & $78 / 855(9.12)$ & $29 / 293(9.90)$ & $3 / 60(5.00)$ & 0.486 \\
PDE4DIP & $82 / 969(8.46)$ & $48 / 666(7.21)$ & $29 / 243(11.93)$ & $5 / 60(8.33)$ & 0.077 \\
CDKN2A & $188 / 2,253(8.34)$ & $112 / 1,411(7.94)$ & $70 / 582(12.03)$ & $6 / 260(2.31)$ & $<0.001$ \\
STK11 & $210 / 2,670(7.87)$ & $191 / 1,964(9.73)$ & $19 / 629(3.02)$ & $0 / 77(0.00)$ & $<0.001$ \\
\hline
\end{tabular}

${ }^{\text {aS }}$ Statistically significant differences were calculated using the Chi-square test to distinguish the mutated-frequency differences between ADC, SCC and SCLC subtypes. ADC, adenocarcinoma; SCC, squamous cell carcinoma; SCLC, small cell lung cancer.

Table II. Number of mutations of driver mutated genes in lung carcinoma with mutated-type and wild-type BAF.

\begin{tabular}{|c|c|c|c|c|c|c|c|c|c|c|c|c|}
\hline \multirow[b]{4}{*}{$\begin{array}{l}\text { Mutated } \\
\text { genes }\end{array}$} & \multicolumn{12}{|c|}{ No. of mutated samples } \\
\hline & \multirow{2}{*}{\multicolumn{2}{|c|}{$\begin{array}{c}\text { Lung } \\
\text { carcinoma, } \mathrm{n}=800\end{array}$}} & \multirow[b]{3}{*}{ P-value ${ }^{a}$} & \multicolumn{9}{|c|}{ Histological type } \\
\hline & & & & \multicolumn{2}{|c|}{$\mathrm{ADC}, \mathrm{n}=582$} & \multirow[b]{2}{*}{ P-value ${ }^{a}$} & \multicolumn{2}{|c|}{$\mathrm{SCC}, \mathrm{n}=177$} & \multirow[b]{2}{*}{ P-value ${ }^{a}$} & \multicolumn{2}{|c|}{ SCLC, $n=41$} & \multirow[b]{2}{*}{ P-value } \\
\hline & $\begin{array}{l}\text { BAF } \\
\text { MT }\end{array}$ & $\begin{array}{l}\text { BAF } \\
\text { WT }\end{array}$ & & $\begin{array}{l}\text { BAF } \\
\text { MT }\end{array}$ & $\begin{array}{l}\text { BAF } \\
\text { WT }\end{array}$ & & $\begin{array}{l}\text { BAF } \\
\text { MT }\end{array}$ & $\begin{array}{l}\text { BAF } \\
\text { WT }\end{array}$ & & $\begin{array}{l}\text { BAF } \\
\text { MT }\end{array}$ & $\begin{array}{l}\text { BAF } \\
\text { WT }\end{array}$ & \\
\hline BAF & 282 & 0 & - & 206 & 0 & - & 67 & 0 & - & 9 & 0 & - \\
\hline TP53 & 161 & 212 & $<0.001$ & 124 & 132 & $<0.001$ & 32 & 51 & 0.857 & 5 & 29 & 0.014 \\
\hline EGFR & 29 & 66 & 0.304 & 26 & 62 & 0.213 & 3 & 4 & 0.781 & 0 & 0 & - \\
\hline KRAS & 65 & 97 & 0.146 & 64 & 95 & 0.133 & 1 & 1 & 0.722 & 0 & 1 & 0.591 \\
\hline MLL3 & 74 & 56 & $<0.001$ & 54 & 41 & $<0.001$ & 18 & 15 & 0.028 & 2 & 0 & 0.006 \\
\hline MLL2 & 41 & 36 & 0.001 & 24 & 17 & 0.001 & 17 & 18 & 0.144 & 0 & 1 & 0.591 \\
\hline ZNF521 & 41 & 37 & 0.001 & 32 & 27 & 0.001 & 8 & 6 & 0.121 & 1 & 4 & 0.910 \\
\hline PDE4DIP & 45 & 36 & $<0.001$ & 29 & 19 & $<0.001$ & 16 & 13 & 0.036 & 0 & 4 & 0.264 \\
\hline CDKN2A & 9 & 12 & 0.460 & 6 & 11 & 0.993 & 3 & 1 & 0.121 & 0 & 0 & - \\
\hline STK11 & 20 & 41 & 0.675 & 20 & 40 & 0.124 & 0 & 1 & 0.434 & 0 & 0 & - \\
\hline
\end{tabular}

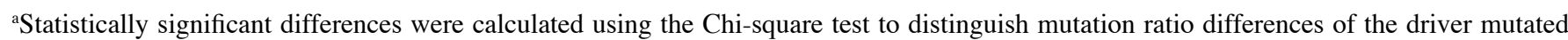
genes in lung cancer samples with mutated-type and wild-type BAF. MT, mutated type; WT, wild-type.

tical difference between mutation rates was determined by the Chi-square test. Pearson correlation analysis was used for investigating the relationship between BAF mutations and overall mutations in lung cancer samples. The statistical analyses were performed using SPSS software. A P-value of $<0.05$ was considered to indicate a statistically significant difference.

\section{Results}

Mutation analysis of BAF complexes in lung carcinoma. The 26 genes encoding mammalian SWI/SNF subunits are listed in Table I. Based on the data from Sanger COSMIC, we first investigated the mutation type and prevalence of these BAF genes in human lung carcinoma samples. All BAF 
Table III. Mutation frequencies of the driver mutated genes in various types of lung carcinoma.

Frequency of mutation, mutated samples/total samples (\%)

\begin{tabular}{lrrrrr} 
& & \multicolumn{3}{c}{ Histological type } & \\
\cline { 2 - 4 } Mutated genes & Lung carcinoma & \multicolumn{1}{c}{ ADC } & \multicolumn{1}{c}{ SCC } & \multicolumn{1}{c}{ SCLC } & P-value ${ }^{\mathrm{a}}$ \\
\cline { 3 - 5 } BAF & $282 / 803(35.12)$ & $206 / 582(35.40)$ & $67 / 177(37.85)$ & $9 / 44(20.45)$ & 0.093 \\
TP53 & $1,423 / 3,142(45.29)$ & $715 / 1,858(38.48)$ & $530 / 1,040(50.96)$ & $178 / 244(72.95)$ & $<0.001$ \\
EGFR & $9,478 / 30,658(30.92)$ & $9,236 / 26,502(34.85)$ & $204 / 3,622(5.63)$ & $38 / 534(7.12)$ & $<0.001$ \\
KRAS & $2,254 / 13,545(16.64)$ & $2,131 / 10,778(19.77)$ & $116 / 2,290(5.07)$ & $7 / 477(1.47)$ & $<0.001$ \\
MLL3 & $133 / 848(15.68)$ & $96 / 609(15.76)$ & $33 / 180(18.33)$ & $4 / 59(6.78)$ & 0.106 \\
MLL2 & $85 / 846(10.05)$ & $44 / 609(7.22)$ & $35 / 180(19.44)$ & $6 / 57(10.53)$ & $<0.001$ \\
ZNF521 & $83 / 851(9.75)$ & $59 / 609(9.69)$ & $14 / 180(7.78)$ & $10 / 62(16.13)$ & 0.160 \\
NF1 & $110 / 1,208(9.11)$ & $78 / 855(9.12)$ & $29 / 293(9.90)$ & $3 / 60(5.00)$ & 0.486 \\
PDE4DIP & $82 / 969(8.46)$ & $48 / 666(7.21)$ & $29 / 243(11.93)$ & $5 / 60(8.33)$ & 0.077 \\
CDKN2A & $188 / 2,253(8.34)$ & $112 / 1,411(7.94)$ & $70 / 582(12.03)$ & $6 / 260(2.31)$ & $<0.001$ \\
STK11 & $210 / 2,670(7.87)$ & $191 / 1,964(9.73)$ & $19 / 629(3.02)$ & $0 / 77(0.00)$ & $<0.001$ \\
\hline
\end{tabular}

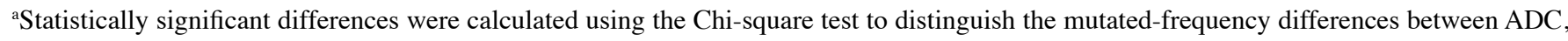
SCC and SCLC subtypes. ADC, adenocarcinoma; SCC, squamous cell carcinoma; SCLC, small cell lung cancer.

Table IV. Mutation numbers of driver mutated genes in lung carcinoma with mutated-type and wild-type BAF.

\begin{tabular}{|c|c|c|c|c|c|c|c|c|c|c|c|c|}
\hline \multirow[b]{4}{*}{$\begin{array}{l}\text { Mutated } \\
\text { genes }\end{array}$} & \multicolumn{12}{|c|}{ No. of mutated samples } \\
\hline & \multirow{2}{*}{\multicolumn{2}{|c|}{$\begin{array}{c}\text { Lung } \\
\text { carcinoma, } \mathrm{n}=800\end{array}$}} & \multirow[b]{3}{*}{ P-value ${ }^{a}$} & \multicolumn{9}{|c|}{ Histological type } \\
\hline & & & & \multicolumn{2}{|c|}{$\mathrm{ADC}, \mathrm{n}=582$} & \multirow[b]{2}{*}{ P-value } & \multicolumn{2}{|c|}{$\mathrm{SCC}, \mathrm{n}=177$} & \multirow[b]{2}{*}{ P-value ${ }^{a}$} & \multicolumn{2}{|c|}{ SCLC, $n=41$} & \multirow[b]{2}{*}{ P-value ${ }^{a}$} \\
\hline & $\begin{array}{l}\text { BAF } \\
\text { MT }\end{array}$ & $\begin{array}{l}\text { BAF } \\
\text { WT }\end{array}$ & & $\begin{array}{l}\text { BAF } \\
\text { MT }\end{array}$ & $\begin{array}{l}\text { BAF } \\
\text { WT }\end{array}$ & & $\begin{array}{l}\text { BAF } \\
\text { MT }\end{array}$ & $\begin{array}{l}\text { BAF } \\
\text { WT }\end{array}$ & & $\begin{array}{l}\text { BAF } \\
\text { MT }\end{array}$ & $\begin{array}{l}\text { BAF } \\
\text { WT }\end{array}$ & \\
\hline BAF & 282 & 0 & - & 206 & 0 & - & 67 & 0 & - & 9 & 0 & - \\
\hline TP53 & 161 & 212 & $<0.001$ & 124 & 132 & $<0.001$ & 32 & 51 & 0.857 & 5 & 29 & 0.014 \\
\hline EGFR & 29 & 66 & 0.304 & 26 & 62 & 0.213 & 3 & 4 & 0.781 & 0 & 0 & - \\
\hline KRAS & 65 & 97 & 0.146 & 64 & 95 & 0.133 & 1 & 1 & 0.722 & 0 & 1 & 0.591 \\
\hline MLL3 & 74 & 56 & $<0.001$ & 54 & 41 & $<0.001$ & 18 & 15 & 0.028 & 2 & 0 & 0.006 \\
\hline MLL2 & 41 & 36 & 0.001 & 24 & 17 & 0.001 & 17 & 18 & 0.144 & 0 & 1 & 0.591 \\
\hline ZNF521 & 41 & 37 & 0.001 & 32 & 27 & 0.001 & 8 & 6 & 0.121 & 1 & 4 & 0.910 \\
\hline PDE4DIP & 45 & 36 & $<0.001$ & 29 & 19 & $<0.001$ & 16 & 13 & 0.036 & 0 & 4 & 0.264 \\
\hline CDKN2A & 9 & 12 & 0.460 & 6 & 11 & 0.993 & 3 & 1 & 0.121 & 0 & 0 & - \\
\hline STK11 & 20 & 41 & 0.675 & 20 & 40 & 0.124 & 0 & 1 & 0.434 & 0 & 0 & - \\
\hline
\end{tabular}

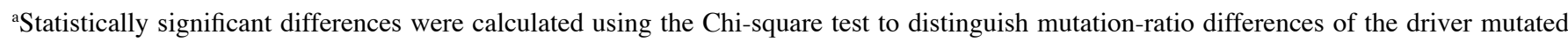
genes in lung cancer samples with mutated-type and wild-type BAF. MT, mutated type; WT, wild-type.

genes except SMARCE1 were mutated to varying degrees (ranging 0.23-7.94\%) in the lung carcinoma samples analyzed (Table II). Of 803 lung carcinoma samples covering all the 26 BAF genes, 282 (35.12\%) exhibited at least 1 mutated BAF gene, and the BAF-mutated frequency indicated no significant difference between various histological types of lung cancer $(\mathrm{P}=0.093$; Table III). These data demonstrated that BAF complexes were frequently mutated, ranking second to
TP53, and played tumor-suppressive roles in lung cancer. To integrate more related information of the lung cancer samples, a mutation profile of BAF complexes was described in these BAF-mutated lung carcinoma samples, where BAF mutation index (BMI), gender, histological types and genotyping of driver genes of lung cancer are shown (Fig. 1). In addition, we also observed mutation ratios of the acknowledged driver genes with frequent mutations in lung cancer, including TP53, 

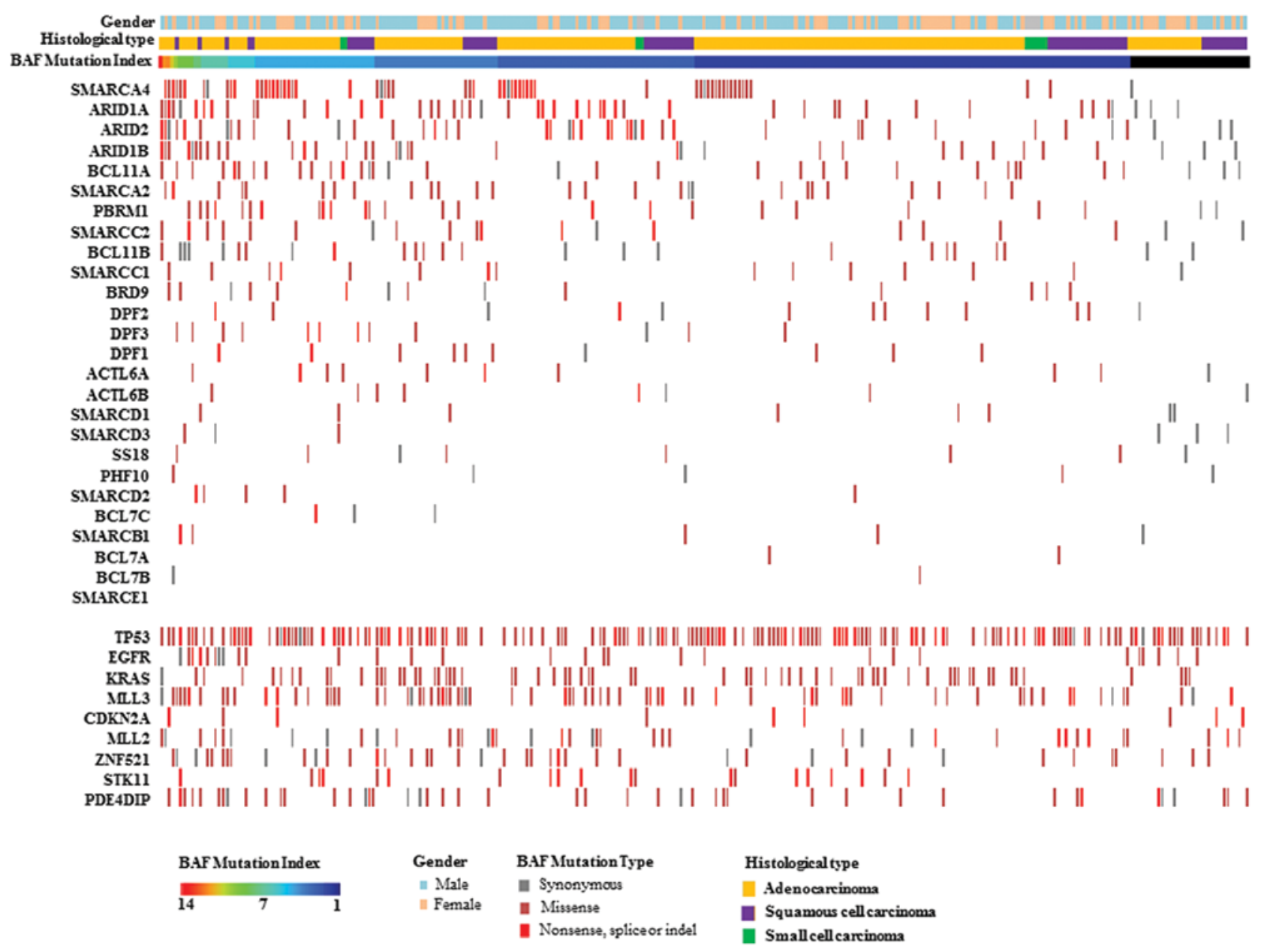

Figure 1. Mutation profile of BAF complexes in lung carcinoma. Each column displays the gender, histological type and BAF mutations in one patient. Each row represents a selected gene of interest, including BAF complex genes and frequently mutated driver genes of lung carcinoma. Cells are colored to denote various mutation types. BAF mutation index (BMI) indicates the accumulative number of BAF complex mutations in each patient, where each type of simple mutation (point mutation, small insertion and deletion) is represented by a specific value; ' 0 ', no mutation; ' 1 ', synonymous mutation; ' 2 ', missense mutation; ' 3 ', nonsense or frameshift mutation.

EGFR, KRAS, MLL3, MLL2, ZNF521, NF1, PDE4DIP, $C D K N 2 A$ and STK11 (Table III). Similar to BAF, the mutation frequencies of $M L L 3, Z N F 521, N F 1$ and PDE4DIP had no significant differences between the 3 histological types of lung carcinoma, implying that these tumor suppressors play a triggering role in the origin of tumor cells, regardless of histological types of lung cancer.

Relationship between BAF complex mutations and genome instability of the lung cancer samples. We next analyzed the numbers of mutated genes and simple mutations occurring in the lung carcinoma samples. Among the 803 lung carcinoma samples analyzed above, 282 BAF-mutated samples significantly exhibited more mutated genes and simple mutations than the BAF wild-type ones (Fig. 2A), which suggests that inactivated mutations of the BAF complexes were associated with genome instability of lung carcinoma. Moreover, we analyzed mutation frequencies of the acknowledged driver mutated genes in these lung carcinoma samples with a mutated-type and wild-type BAF genotype. We analyzed the available genotyping information of the top-10 driver mutated genes from the 803 lung carcinoma samples, 800 of which covering 9 driver mutated genes (no coverage with NF1) were available for further analysis. Significantly, TP53, MLL3, MLL2, ZNF521 and PDE4DIP were more often mutated in the BAF-mutated samples than the BAF wild-type ones (Table IV). According to BAF-mutated extents, these BAF-mutated lung carcinoma samples were categorized into two groups: one group with $\mathrm{BMI} \geq 3$, the other with BMI $\leq 2$. The group with BMI $\geq 3$ had more significant mutated genes and simple mutations than the other group with BMI $\leq 2$ (Fig. 2B). To further establish the relationship between BAF complex mutations and genome instability, we correlated the BMI with the number of overall mutations. Pearson correlation analysis showed that a positive correlation existed between the BMI and the numbers of mutated genes $\left(\mathrm{R}^{2}=0.718, \mathrm{P}<0.001\right)$ or simple mutations $\left(\mathrm{R}^{2}=0.682, \mathrm{P}<0.001\right)$ (Fig. 2C). Collectively, these data suggest that the BAF complex mutations are linked with genomic instability of lung cancer types.

Next, we further investigated the overall mutation differences between the mutated-type and wild-type genotyping of each individual BAF gene in the lung carcinoma samples, respectively. More mutated genes or simple mutations significantly appeared in the lung carcinoma sample with 

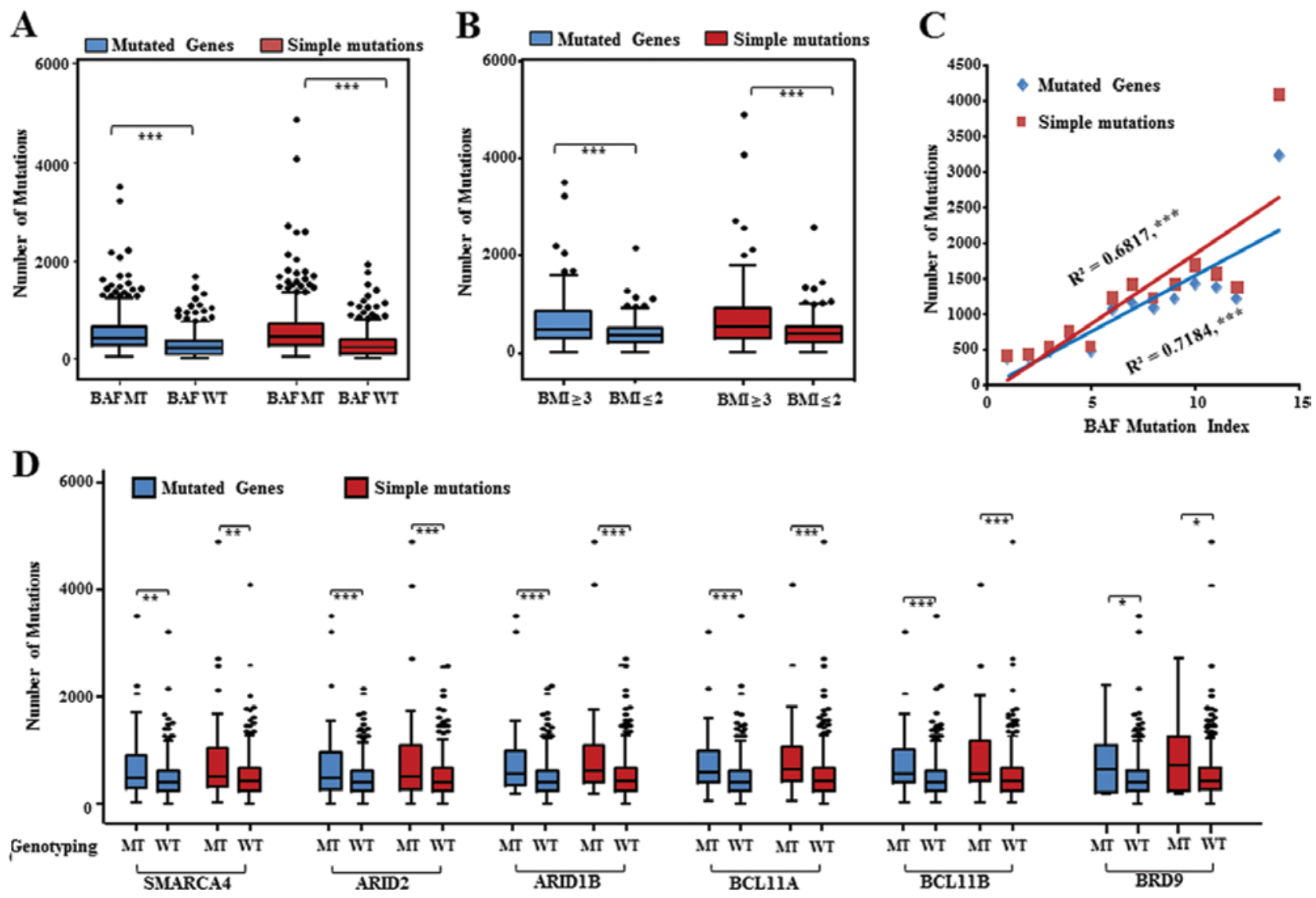

Figure 2. BAF complex mutations are associated with genome instability of lung cancer. (A and B) Tukey box-and-whiskers plot indicates the numbers of mutated genes and simple mutations occurring in these lung cancer samples as analyzed, which were grouped into (A) BAF mutated-type (MT) and BAF wild-type (WT) or (B) BMI $\geq 3$ and BMI $\leq 2$, according to the BAF complex genotyping or BAF mutation index (BMI), respectively. A two-tailed t-test was used to determine statistical significance. (C) Pearson correlation analysis was performed between the BMI and the numbers of mutated genes or simple mutations in lung carcinoma samples analyzed. (D) Tukey box-and-whiskers plot shows the numbers of mutated genes and simple mutations occurring in these lung cancer samples analyzed, according to mutated-type (MT) and wild-type (WT) genotyping of 6 genes: SMARCA4, ARID2, ARID1B, BCL11A, BCL11B and $B R D 9$. Statistical analyses were performed with a two-tailed t-test. ${ }^{*} \mathrm{P}<0.05 ;{ }^{* *} \mathrm{P}<0.01 ;{ }^{* * * *} \mathrm{P}<0.001$.

the mutated-type genotyping of 6 genes, SMARCA4, ARID2, $A R I D 1 B, B C L 11 A, B C L 11 B$ and BRD9 (Fig. 2D). There were no significant differences between the mutated-type and wildtype genotyping of the other BAF genes in the lung carcinoma samples (data not shown). Accordingly, the 6 BAF genes primarily contribute to safeguard genome stability against mutation occurrence.

Establishment of mutation reporters of lung cancer cell lines. To evaluate the mutation ratio in vitro, we developed a mutation reporter of lung cancer cells. First, a slippage vector construct contains $17 \mathrm{CA}$-dinucleotide repeats cloned just downstream from the initiation codon of the luciferase gene, which leads to the luciferase-coding sequence out of frame. However, genome instability resulting in gains of one or loss of two CA repeats will restore the reading frame (Fig. 3A). Then the linearized slippage vector containing the hygromycin resistance gene for selection of stable cell lines was transfected into Calu-3 cells, a lung adenocarcinoma cell line. We cultured Calu-3 cells with selective medium every 3-4 days until hygromycin-resistant colonies were visible, and the 12 colonies were selected for further identification. The results showed that both target fragments of the CMV promoter and Luc element on the slippage vector could be amplified by designed primers from genomic
DNA of the 12 colonies. To evaluate the transcript levels of the slippage-Luc, the same primers were used to amplify cDNA of the 12 cell sublines using RT-PCR. The slippage-Luc transcripts were detected by the primers for Luc element in 4 of the 12 cell colonies, where 2 colonies (H10, H12) exhibiting obvious slippage-Luc transcripts were selected for mutation reporters (Fig. 3B).

SMARCA4 knockdown leads to an increased mutation ratio. To confirm the role of BAF inactivation in genome stability in vitro, we selected the most frequently mutated BAF gene, SMARCA4, for the mutation ratio assay as loss of function in the established mutation reporters of Calu-3 cells without SMARCA4 mutations found (data not shown). pSUPER constructs containing shRNA (sh1, sh2, sh3 and sh4) against SMARCA4 were transfected into the two established Calu-3 cell sublines (H10 and H12), respectively. Three days after transfection, we evaluated the RNAi knockdown efficiency of these shRNAs, using quantitative RT-PCR assay. The result showed that the mRNA levels of SMARCA4 were efficiently and significantly decreased to $<50 \%$ by sh-1 and sh-3, as compared to shNC (Fig. 4A). Subsequently, we screened G418-resistant colonies to establish double-stable sublines with SMARCA4 downregulation, based upon the 


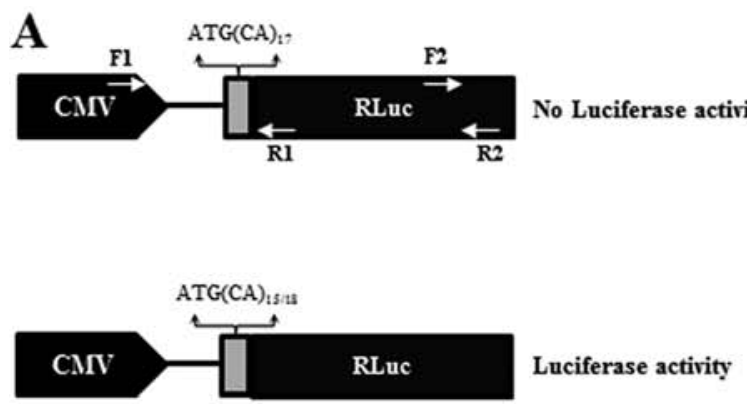

$\begin{array}{lllllllllllllll}\text { B } & \text { gDNA } & \text { H1 } & \text { H2 } & \text { H3 } & \text { H4 } & \text { H5 } & \text { H6 } & \text { H7 } & \text { H8 } & \text { H9 } & \text { H10 } & \text { Hll } & \mathrm{H}_{2} & \mathrm{H}_{2} \mathrm{O}\end{array}$

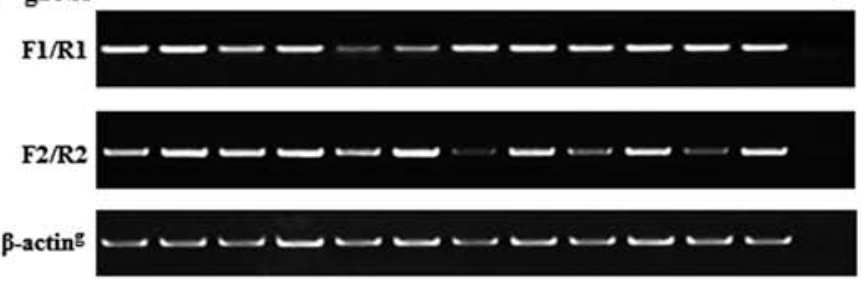

$\begin{array}{llllllllllllllllll}\text { cDNA } & \mathrm{Hl} & \mathrm{H} 2 & \mathrm{H} 3 & \mathrm{H} 4 & \mathrm{H} 5 & \mathrm{H} 6 & \mathrm{H} 7 & \mathrm{H} 8 & \mathrm{H} 9 & \mathrm{H} 10 & \mathrm{H} 11 & \mathrm{Hl} & \mathrm{H} & \mathrm{H} & \mathrm{O}\end{array}$

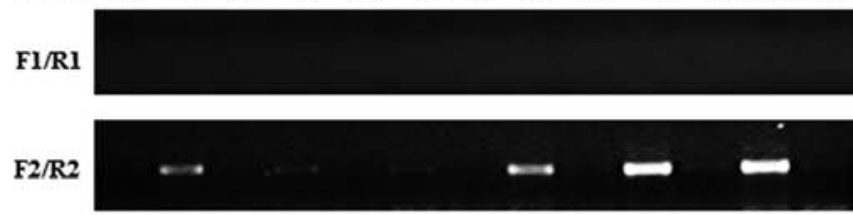

$\beta$-actinc

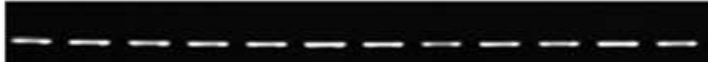

Figure 3. Mutation reporters with slippage-luciferase transcripts were established in a lung adenocarcinoma cell line. (A) A slippage-luciferase construct used to evaluate the mutation ratio through luciferase activity assay is shown, where the slippage vector construct contains 17 CA-dinucleotide repeats cloned just downstream from the initiation codon of the luciferase gene, which leads to the luciferase-coding sequence out of frame (upper) and genome instability resulting in gains of one or loss of two CA repeats will restore the reading frame (lower). Two pairs of primers for the CMV promoter and luciferase element of the slippage vector are indicated by corresponding arrows. (B) Twelve selected hygromycin-resistant colonies were subject to PCR identification, and their genomic DNA and cDNA were amplified by the indicated primers, respectively, where $\beta$-actin ${ }^{\mathrm{g}}$ for genomic DNA and $\beta$-actin ${ }^{\mathrm{c}}$ for cDNA were used as loading controls.

A

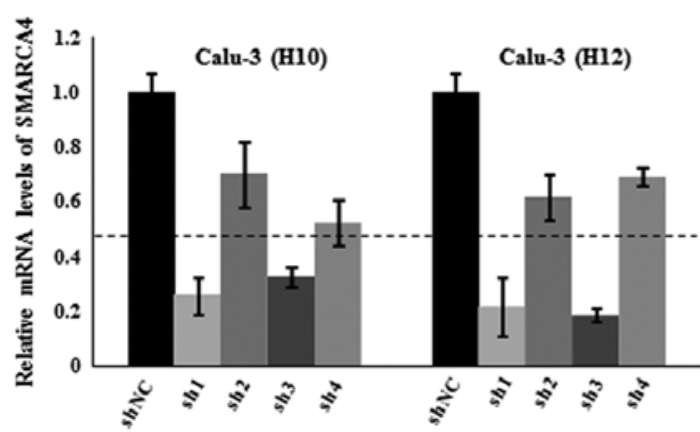

B
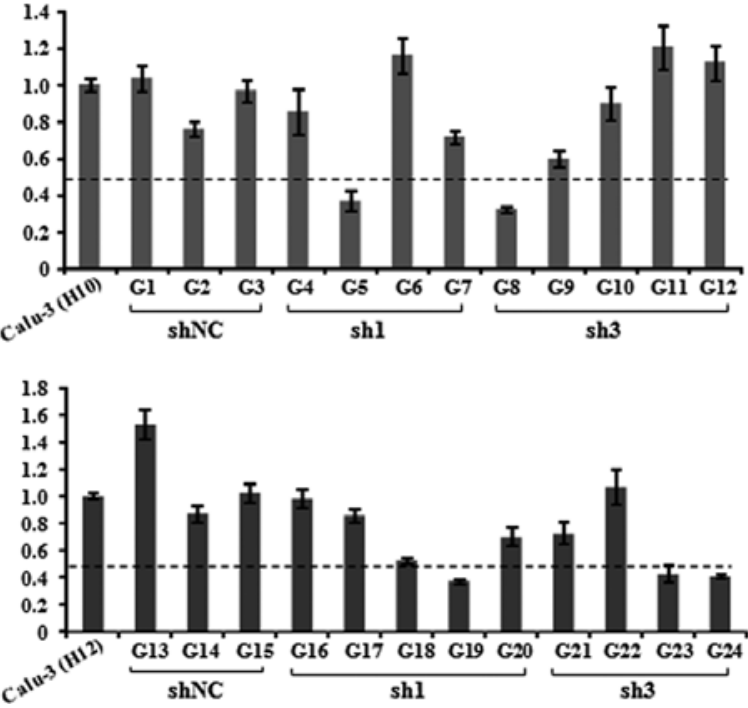

C

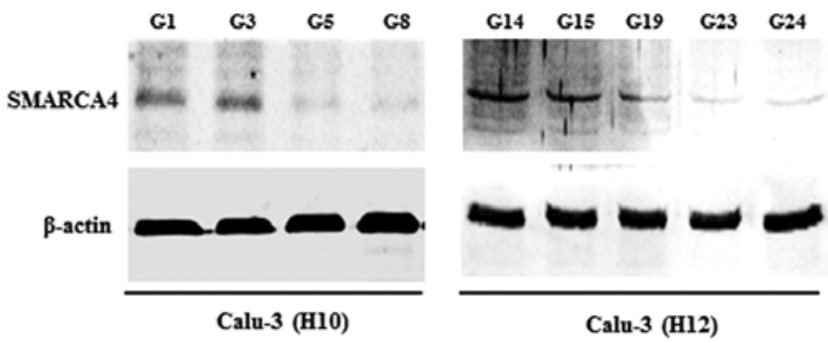

D

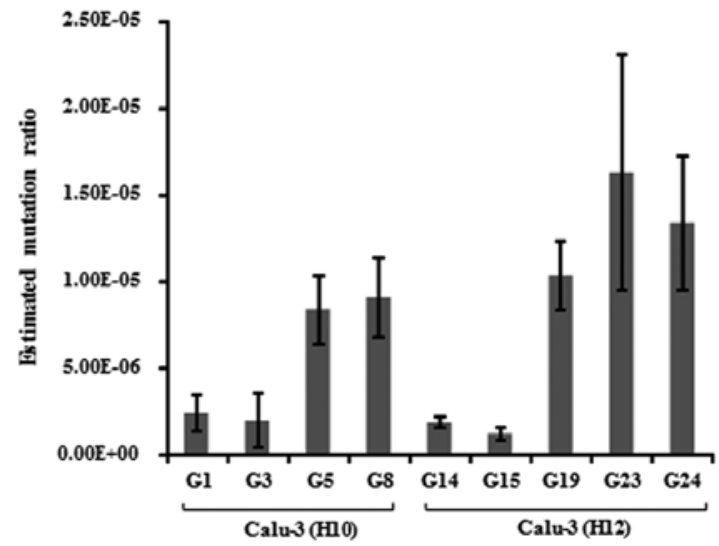

Figure 4. SMARCA4 knockdown leads to increased mutation incidence. (A) Efficiency of the pSUPER-mediated SMARCA4 knockdown was assessed using real-time PCR in the two Calu-3 cell sublines, H10 and H12. (B) SMARCA4 expression was evaluated by real-time PCR in these 24 double-stable Calu-3 cell colonies stably transfected with pSUPER constructs carrying shNC, sh1 and sh3, respectively. (C) SMARCA4 protein expression was examined by western blot assay in these double-stable Calu-3 cell sublines, where $\beta$-actin was used as a loading control. (D) Mutation rates of reporter cells with stable SMARCA4 knockdown (G5, G8, G19, G23 and G24) were determined by Luria-Delbrück fluctuation analyses. As controls, reporter cells were transfected with the pSUPER vector carrying shNC (G1, G3, G14 and G15). 
two Calu-3 cell reporters H10 and H12. Of these double-stable colonies, 5 colonies (G5, G8, G19, G23 and G24) with $<50 \%$ SMARCA4 expression levels compared with their progenitor cells and 4 colonies (G1, G3, G14 and G15) close to the original SMARCA4 levels in their progenitor cells were used for mutation ratio analysis (Fig. 4B). Furthermore, we performed western blot assays to examine SMARCA4 protein expression in these selected double-stable cells. The SMARCA4 protein level was markedly decreased in the 5 cell sublines with stable SMARCA4 knockdown, as compared with the controls with shNC in the two reporter cell lines (H10 and H12), respectively (Fig. 4C). These reporter cells were expanded for 3 weeks, where luciferase activity was measured and Luria-Delbrück fluctuation analyses were then used to calculate the mutation rate. Upon the pSUPER-mediated SMARCA4 knockdown, the mutation ratios were significantly increased in the mutation reporter cells (Fig. 4D). Collectively, these observations suggest that SMARCA4 knockdown may lead to the increased mutations in human lung cancer.

\section{Discussion}

In the present study, we described the mutation incidence of SWI/SNF chromatin remodeling genes in human lung carcinoma, and then explored the genetic features of the BAF-mutated lung cancer samples. As expected, our analysis showed that all BAF genes except SMARCE1 were mutated in these lung carcinoma samples, $35.12 \%$ of which exhibited at least 1 mutated BAF gene, ranking second to TP53 mutation incidence. We found that TP53, EGFR, KRAS, MLL2, $C D K N 2 A$ and STK11 had significant differences in mutation frequency among adenocarcinoma, squamous cell carcinoma and small cell lung cancer, basically consistent with previous reports (19-21). However, it is worth mentioning that the mutation frequency of BAF complexes had no significant difference between the 3 histological types of human lung carcinoma, implying that BAF complexes extensively underwent genetic damage in lung carcinoma, regardless of histological types. In addition, the TP53 mutation is associated with smoking-induced lung cancer. Smokers with lung cancer have a higher risk of TP53 mutation than non-smokers (22). However, our current data shed little light on the question of whether SWI/SNF mutations are associated with smokers in lung cancer.

The SWI/SNF chromatin remodeling complex plays essential roles in a wide variety of cellular and biological processes including gene expression, nuclear organization, centromere function, chromosomal stability, differentiation, proliferation and DNA repair (23-29). Nevertheless, how these inactivated mutations in these complexes contribute to tumor suppression is unclear. Notably, our analysis revealed that BAF complex mutations were associated with genome instability in lung carcinoma, which conforms to the biological roles of the SWI/SNF complex in chromosomal stability and DNA repair. Among these BAF genes, SMARCA4, ARID2, ARIDIB, $B C L 11 A, B C L 11 B$ and $B R D 9$ are possible major contributors to genome stability and/or DNA repair. To elucidate this issue, we established a mutation reporter to evaluate the mutation ratio in a lung carcinoma cell line, Calu-3. SMARCA4, the most frequently mutated BAF gene in lung carcinoma $(8,12,30)$, was used from loss of function assay in established mutation reporter. We confirmed that the SMARCA4 gene protected the genome against mutation occurrence, upon stable SMARCA4 in mutation reporter cells of lung carcinoma, consistent with its requirement during embryogenesis and its role as a tumor suppressor to maintain genome stability (31).

In conclusion, inactivated mutations of BAF complexes are significantly associated with more overall mutations in lung carcinoma, and BAF complexes play an important role in maintaining the genome stability of lung cancer.

\section{Acknowledgements}

The present study was funded by The First Affiliated Hospital of Soochow University.

\section{References}

1. Saika K and Sobue T: Cancer statistics in the world. Gan To Kagaku Ryoho 40: 2475-2480, 2013 (In Japanese).

2. Malvezzi M, Bertuccio P, Levi F, La Vecchia C and Negri E: European cancer mortality predictions for the year 2013. Ann Oncol 24: 792-800, 2013.

3. Yang G, Wang Y, Zeng Y, et al: Rapid health transition in China, 1990-2010: findings from the Global Burden of Disease Study 2010. Lancet 381: 1987-2015, 2013.

4. She J, Yang P, Hong Q and Bai C: Lung cancer in China: challenges and interventions. Chest 143: 1117-1126, 2013.

5. Son JW: Year-in-Review of Lung Cancer. Tuberculosis and respiratory diseases 73: 137-142, 2012.

6. Agullo-Ortuno MT, Lopez-Rios F and Paz-Ares L: Lung cancer genomic signatures. J Thorac Oncol 5: 1673-1691, 2010.

7. Wilson BG and Roberts CW: SWI/SNF nucleosome remodellers and cancer. Nat Rev Cancer 11: 481-492, 2011.

8. Oike T, Ogiwara H, Nakano T, Yokota J and Kohno T: Inactivating mutations in SWI/SNF chromatin remodeling genes in human cancer. Jpn J Clin Oncol 43: 849-855, 2013.

9. Reisman D, Glaros S and Thompson EA: The SWI/SNF complex and cancer. Oncogene 28: 1653-1668, 2009.

10. Manceau G, Letouze E, Guichard C, et al: Recurrent inactivating mutations of ARID2 in non-small cell lung carcinoma. Int $\mathbf{J}$ Cancer 132: 2217-2221, 2013.

11. Jones S, Li M, Parsons DW, et al: Somatic mutations in the chromatin remodeling gene ARID1A occur in several tumor types. Human Mutat 33: 100-103, 2012.

12. Rodriguez-Nieto S, Cañada A, Pros E, et al: Massive parallel DNA pyrosequencing analysis of the tumor suppressor BRG1/SMARCA4 in lung primary tumors. Hum Mutat 32: E1999-E2017, 2011.

13. Ho L, Ronan JL, Wu J, et al: An embryonic stem cell chromatin remodeling complex, esBAF, is essential for embryonic stem cell self-renewal and pluripotency. Proc Natl Acad Sci USA 106: 5181-5186, 2009.

14. Kadoch C, Hargreaves DC, Hodges C, et al: Proteomic and bioinformatic analysis of mammalian SWI/SNF complexes identifies extensive roles in human malignancy. Nat Genet 45: 592-601, 2013.

15. Forbes SA, Bindal N, Bamford S, et al: COSMIC: mining complete cancer genomes in the Catalogue of Somatic Mutations in Cancer. Nucleic Acids Res 39: D945-D950, 2011.

16. Bamford S, Dawson E, Forbes S, et al: The COSMIC (Catalogue of Somatic Mutations in Cancer) database and website. Br J Cancer 91: 355-358, 2004.

17. Koziol JA: A note on efficient estimation of mutation rates using Luria-Delbrück fluctuation analysis. Mutat Res 249: 275-280, 1991.

18. Hall BM, Ma CX, Liang P and Singh KK: Fluctuation analysis calculator: a web tool for the determination of mutation rate using Luria-Delbruck fluctuation analysis. Bioinformatics 25: $1564-1565,2009$

19. Wistuba, II, Gazdar AF and Minna JD: Molecular genetics of small cell lung carcinoma. Semin Oncol 28: 3-13, 2001. 
20. Takahashi T, Suzuki H, Hida T, Sekido Y, Ariyoshi Y and Ueda R: The p53 gene is very frequently mutated in small-cell lung cancer with a distinct nucleotide substitution pattern. Oncogene 6: 1775-1778, 1991.

21. Dearden S, Stevens J, Wu YL and Blowers D: Mutation incidence and coincidence in non small-cell lung cancer: meta-analyses by ethnicity and histology (mutMap). Ann Oncol 24: 2371-2376, 2013.

22. Liu X, Lin XJ, Wang CP, et al: Association between smoking and p53 mutation in lung cancer: a meta-analysis. Clin Oncol (R Coll Radiol) 26: 18-24, 2014

23. de la Serna IL, Carlson KA and Imbalzano AN: Mammalian SWI/SNF complexes promote MyoD-mediated muscle differentiation. Nat Genet 27: 187-190, 2001

24. Watanabe H, Mizutani T, Haraguchi T, et al: SWI/SNF complex is essential for NRSF-mediated suppression of neuronal genes in human nonsmall cell lung carcinoma cell lines. Oncogene 25 470-479, 2006

25. Vradii D, Wagner S, Doan DN, et al: Brg1, the ATPase subunit of the SWI/SNF chromatin remodeling complex, is required for myeloid differentiation to granulocytes. J Cell Physiol 206: $112-118,2006$
26. Romero OA and Sanchez-Cespedes M: The SWI/SNF genetic blockade: effects in cell differentiation, cancer and developmental diseases. Oncogene 33: 2681-2689 2014.

27. Euskirchen GM, Auerbach RK, Davidov E, et al: Diverse roles and interactions of the SWI/SNF chromatin remodeling complex revealed using global approaches. PLoS Genet 7: e1002008, 2011.

28. Kasten MM, Clapier CR and Cairns BR: SnapShot: chromatin remodeling: SWI/SNF. Cell 144: 310.e1, 2011.

29. Xu Y and Price BD: Chromatin dynamics and the repair of DNA double strand breaks. Cell Cycle 10: 261-267, 2011.

30. Matsubara D, Kishaba Y, Ishikawa S, et al: Lung cancer with loss of BRG1/BRM, shows epithelial mesenchymal transition phenotype and distinct histologic and genetic features. Cancer Sci 104: 266-273, 2013.

31. Cohen SM, Chastain PD II, Rosson GB, et al: BRG1 co-localizes with DNA replication factors and is required for efficient replication fork progression. Nucleic Acids Res 38: 6906-6919, 2010. 